(C) 1996 IEEE. Personal use of this material is permitted. However, permission to reprint/republish this material

for advertising or promotional purposes or for creating new collective works for resale or redistribution to servers

or lists, or to reuse any copyrighted component of this work in other works must be obtained from the IEEE.

\title{
Status of the Nuclotron Slow Extraction System
}

\author{
V.I.Chernikov, I.B.Issinsky, O.S.Kozlov, V.A.Mikhailov, S.A.Novikov \\ 141980, Laboratory of High Energies \\ Joint Institute for Nuclear Research, Dubna, Russia
}

\begin{abstract}
A general description of the slow exraction system of a charged particle beam from the Nuclotron is presented. The third integer resonance is used for the slow step-over extraction mechanism. The septum deflectors, that are an electrostatic septum and two superconducting Lambertson magnets, are the most complicated components of the Nuclotron slow extraction system. A detailed design of these elements based on computer simulation is considered too. The septum deflectors are under construction and bench testing before their placement in the Nuclotron ring.
\end{abstract}

\section{Introduction}

The Nuclotron[1], [2], a superconducting synchrotron, is intended to accelerate nuclei and multicharged ions (up to uranium) from an injection energy of $5 \mathrm{MeV} / \mathrm{u}$ up to an energy of 6 $\mathrm{GeV} / \mathrm{u}$ for the charge to mass ratio $\mathrm{Z} / \mathrm{A}=0.5$.

The Nuclotron is a strong-focusing separated function synchrotron with a circumference of $251.52 \mathrm{~m}$. It has 32 FODO cells separated into 8 structural superperiods. The magnetic ring contains 96 SC-dipoles and 64 SC-quadrupoles. The Nuclotron is placed on the ground floor of the Synchrophasotron building, $3.76 \mathrm{~m}$ down relative to the linac and the transport beam line plane. A scheme of the Nuclotron superperiod and a layout of the slow extraction elements over the Nuclotron ring are shown in Fig.1.

The Nuclotron was put into commissioning in 1993. Now its cryogenic, power supply and magnet protecting systems are under development to raise their operation stability and to reach reliable work at the projected energy. Limited financing does not allow one to carry out the full program of putting the accelerator into exploitation. A stable circulation and acceleration of deutrons up to $2.5 \mathrm{GeV} / \mathrm{u}$ at a beam intensity of $2 \times 10^{9} \mathrm{ppp}$ were obtained in December 1994. The first physics experiments with an internal target were carried out. The next run is scheduled to begin in the late May of 1995.

\section{Slow extraction mechanism}

In accordance with the chosen betatron frequences $\mathrm{Q}_{x} \simeq 6.8$, $\mathrm{Q}_{z} \simeq 6.85$, it is optimum to use the third integer nonlinear resonance $3 \mathrm{Q}_{x}=20$ for slow extraction[3]. The operating point is moved to the resonance band by the structural quadrupoles. At this time the 20-th harmonic of sextupole nonlinearity is excited by 4 slow extraction sextupole lenses S1-S4 located in the minima of the dispertion function. 4 slow extraction quadrupole lenses Q1-Q4 are uniformly placed over the Nuclotron ring to produce the coherent betatron tune shift $\Delta \mathrm{Q}_{x}$ within the resonance sideband and to prevent excitation of the parametric resonances $2 \mathrm{Q}_{x, z}=13,2 \mathrm{Q}_{x, z}=14$.

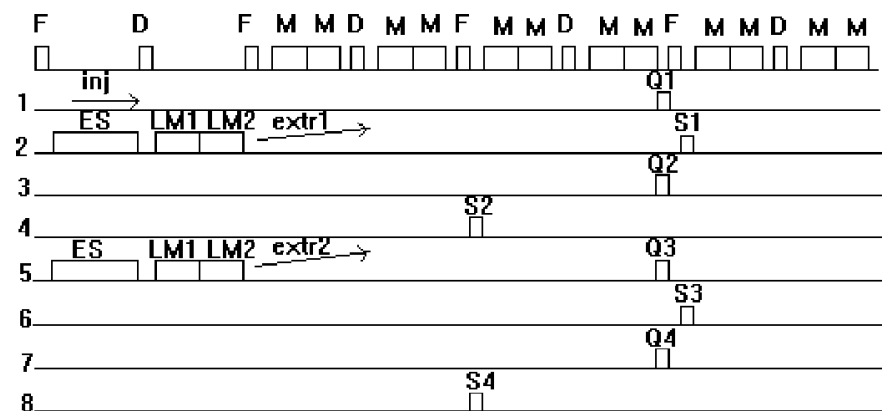

Figure. 1. Scheme of the Nuclotron superperiod and layout of the slow extraction elements over the Nuclotron ring

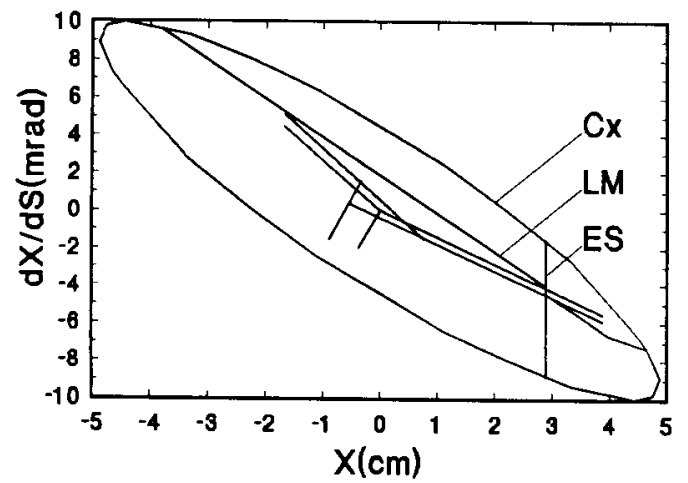

Figure. 2. Phase picture at the ES entrance @ $6 \mathrm{GeV} / \mathrm{u}$

The beam will be extracted in two directions to be used in two experimental areas by means of the electrostatic septum (ES) and the two-section Lambertson magnet (LM) placed in the long drift spaces of the second and fifth superperiods. The ES deflects particles in the horizontal plane. The LM allows one to bend the beam in the vertical plane in the direction of the experimental halls and to pass over the lens body situated at the end of the staight section. General parameters of the slow extraction system are given in Table 2 .

The phase trajectories at the entrance of the ES for an energy of $6 \mathrm{GeV} / \mathrm{u}$ are shown in Fig.2. There are an ellipse surrounding the Nuclotron acceptance $\left(\mathrm{C}_{x}=210 \mathrm{~m} \pi \mathrm{mm}\right.$ rad $)$ and phase portraits for the electrostatic septum and Lambertson magnet. The momentum spread changing during the slow extraction process is shown in Fig.3.

\section{Electrostatic septum}

The efficiency of slow extraction strongly depends on the thickness of the first extraction septum as compared to the growth of the resonant betatron amplitudes in the final turns before extraction. A $95 \%$ efficiency of extraction will be realized 


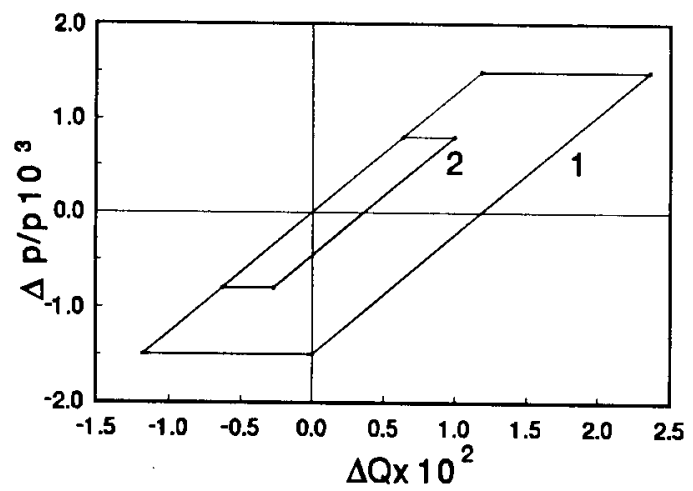

Figure. 3. Momentum spread changing during slow extraction: 1-@200 MeV/u, 2-@6 GeV/u

by a very aligned electrostatic septum formed from grounded $0.1 \mathrm{~mm}$ tungsten-renium wires with an effective thickness of $0.3 \mathrm{~mm}$ and a spacing of $1 \mathrm{~mm}$ that is the anode. The effective length of the parallel electrodes, cathode and anode, is 3 $\mathrm{m}$. The wire anode separates the region of the circulating beam from the region where there exists an electrostatic field and has a ground potential. The cathode is at a high negative potential of up to $200 \mathrm{kV}$. A gap of $2 \mathrm{~cm}$ with a field of $100 \mathrm{kV} / \mathrm{cm}$ is needed to reach a required deflection of about $2 \mathrm{mrad}$. The cross section of the electrostatic deflector is shown in Fig. 4 where the coordinates are connected to the Nuclotron equilibrium orbit.

The calculations were aimed to form the cathode surface for the required uniformity of the deflecting field no less than $97 \%$ in the working region $(3 \mathrm{~cm} \leq \mathrm{x} \leq 5 \mathrm{~cm},-2 \mathrm{~cm} \leq \mathrm{z} \leq 2 \mathrm{~cm})$ where the extracted beam passes through the gap. The nonuniformity and distribution of the electrostatic field are given in Figs.4,5.

There are some arguments in favour of a tungsten-renium wire anode. It is pretty stable under sparking when the energy available in the discharge releases. It is easier to build and to

Table I

General characteristics of the Nuclotron slow extraction system

\begin{tabular}{|l||c|}
\hline Kinetic ion energy (Z/A=0.5) & $0.2 / 6 \mathrm{GeV} / \mathrm{u}$ \\
\hline Effective length of quadrupoles & $0.3 \mathrm{~m}$ \\
Effective length of sextupoles & $0.3 \mathrm{~m}$ \\
$\mathrm{~dB}_{z} / \mathrm{dx}$ (quadrupoles) & $0.14 / 0.68 \mathrm{~T} / \mathrm{m}$ \\
$\mathrm{d}^{2} \mathrm{~B}_{z} / \mathrm{dx}^{2}$ (sextupoles ) & $13.3 / 233 \mathrm{~T} / \mathrm{m}^{2}$ \\
Effective length of ES & $3.3 / 3.3 \mathrm{~m}$ \\
Effective thickness of ES & $0.42 / 0.31 \mathrm{~mm}$ \\
Electric field tension in ES & $1.26 / 10 \mathrm{MV} / \mathrm{m}$ \\
Effective length of LM1 and LM2 & $1.5 \mathrm{~m}$ \\
Field in LM1 & $0.1 / 1.1 \mathrm{~T}$ \\
Field in LM2 & $0.15 / 1.6 \mathrm{~T}$ \\
Effective emittance of the extracted & $9.5 / 2.5 \pi \mathrm{mm} \mathrm{mr}$, \\
beam: horizontal, vertical & $20 / 2.0 \pi \mathrm{mm} \mathrm{mr}$ \\
Instantaneous momentum spread & $(7 / 0.2) \times 10^{-3}$ \\
Duration & $(1-10) \mathrm{sec}$ \\
Duty factor of the extracted current & $95 / 95 \%$ \\
Slow extraction efficiency & $95 / 96 \%$ \\
\hline
\end{tabular}

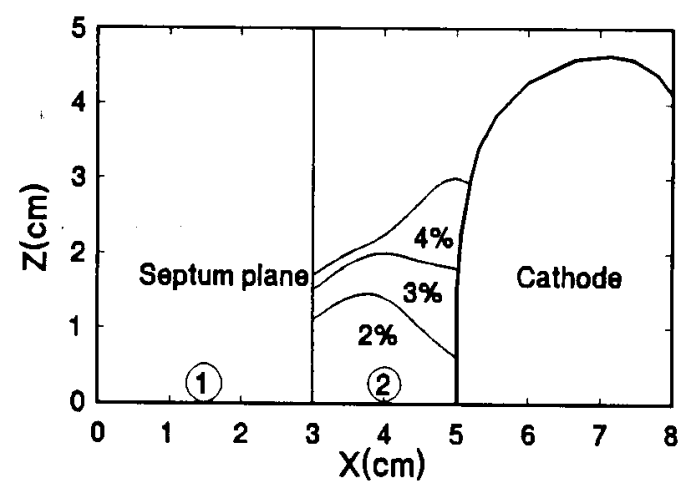

Figure. 4. Scheme of the ES (half) and regions of nonuniformity of the electrostatic field: 1-circulating, 2-extracted beams

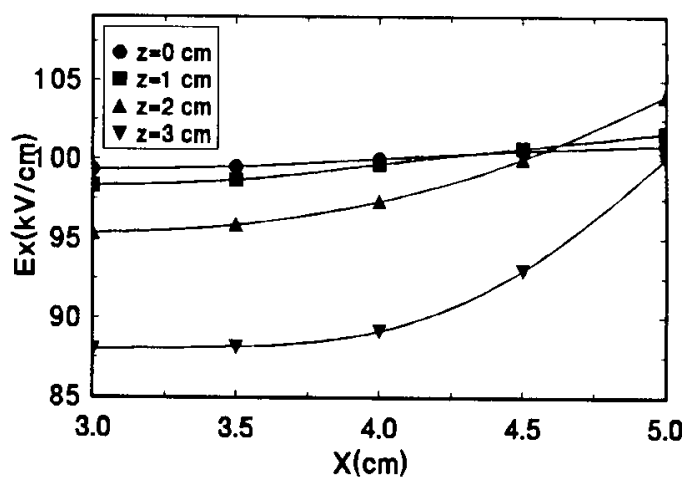

Figure. 5. Distribution of the electrostatic field in the ES gap

maintain a perfect plane with an array of wires than foil. The mean Coulomb scattering angle of particles hitting the septum is smaller and thus the losses are smaller. The oxide-coated titanium cathode can stand a higher energy in the sparks across a small gap. The calculated overtension on the cathode surface reaches $6 \%$ that is within the admissible boundary. Of course, the insulation of the ES gap will greatly depend on cleanliness of the vacuum system and advanced conditioning of the electrodes.

\section{Lambertson magnets}

LM1[5] and LM2 are $1.5 \mathrm{~m}$ long magnetic deflectors with superconducting windings whose transverse sections are shown in Fig.6. Two apertures in the yoke for circulating and extracted beams are separated by 3 and $7 \mathrm{~mm}$ septa. They deflect the extracted beam up at an angle of $80-110 \mathrm{mrad}$ to by-pass the following quadrupole lens and to rise the beam to the level of the existing transport channels. The maximun magnetic fields in LM1 and LM2 are 1.2 T and 1.8 T, respectively.

The two-dimensional designs[4] of the magnetic yoke and coil parameters were aimed first of all to reduce the leakage field in the circulating beam region and then to gain a good uniformity of the working field. The essential cut-out in the LM2 extracted beam aperture in front of the magnetic septum is designed for extra reduction of the leakage field. As the calculations show, the nonuniformity of the working field does not exceed $1-2 \%$ that is satisfactory for a single beam passing over the 

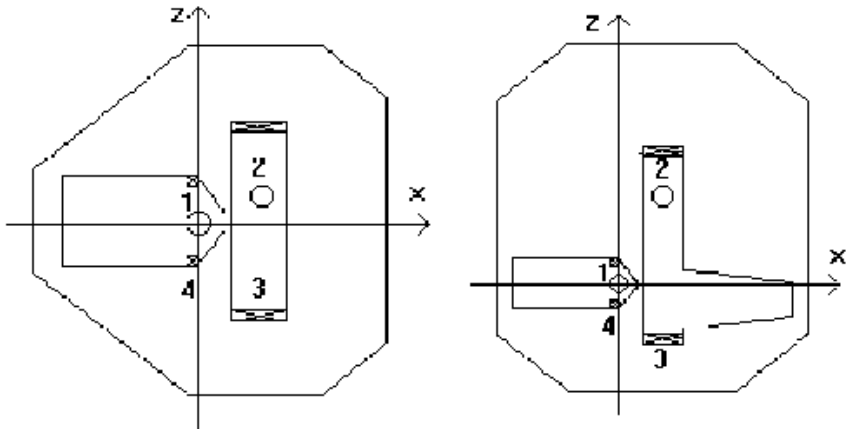

Figure. 6. Scheme of the Lambertson magnets: 1-circulating, 2-extracted beams, 3-main coils, 4-current coils

extracting region. But with a compact magnetic yoke and thin septa we shall have anyway a leakage field of up to $2-3 \%$ of the main one at a beam energy of 5-6 GeV/u due to the saturation of the magnetic yoke. That is inadmissible because of considerable closed orbit distortions, during the acceleration process. Since the $\mathrm{B}_{x}$-component of the leakage field is much greater than the $\mathrm{B}_{z}$-component, the $\mathrm{z}$-distortion will be much greater (up to $\pm 20 \mathrm{~mm}$ ) than the $\mathrm{x}$-distortion (up to $\pm 2 \mathrm{~mm}$ ). To overcome this, one can set out current coils in the LM1,2 circulating beam aperture for independent correction of the leakage field. The calculations show that one can suppress leakage fields and provide the $\mathrm{x}, \mathrm{z}$-distortions over a range of $\pm 3 \mathrm{~mm}$ using only one coil (Fig.6).

\section{Conclusion}

Commissioning the Nuclotron, a new superconducting synchrotron at the Lab of High Energies, JINR is being continued. The machine development program is directed to the extraction of accelerated beams. The construction of the Nuclotron slow extraction system presented in this paper is under way. Nowadays the superconducting slow extraction quadrupoles and sextupoles are being arranged in the Nuclotron ring. The ES anode and cathode are under construction. The first bench testing of LM1[5] has already been carried out. Cooling down to 4.6 $\mathrm{K}$, control of the cryogenic temperature and magnetic measurements have been performed. After the next LM2 magnetic field measurements and placing LM1 and LM2 in the ring, the slow extraction without ES will be realized despite of a small effectiveness of about $10-15 \%$.

\section{References}

[1] A.M.Baldin et al. Nuclotron Status Report, in IEEE Trans. on Nucl. Sci., NS-30, pp.3247-3249, March 1983.

[2] I.B.Issinsky et al. Nuclotron Lattice, EPAC, Nice, June 1216, 1990, vol.1, p.458-460.

[3] B.V.Vasilishin et al. The Scheme and Main Parameters of the Nuclotron Slow Extraction System. Preprint JINR, 986-511, Dubna, 1986.

[4] R.F.Holsinger, Ch.Iselin. The CERN-POISSON Program Package (POISCR) User Guide. CERN/TH, 1983.
[5] V.N.Buldakovsky et al. Nuclotron Slow Extraction Lambertson Magnet. Proc. of the XIII Conf. on Particle Accelerators, Dubna, 1992. 